\title{
How to take a comfortable and energetic bath?
}

\author{
Qi Cao
}

North China Electric Power University, Baoding 071000, China

15176275778@163.com

Keywords: the temperature of the bathtub water in time,the optimal analysis method

\begin{abstract}
Firstly we build a set of models to describe the temperature of the bathtub water in time. In this section, we analyze the variation process of water temperature under the condition of Natural cooling and injecting hot water respectively through a macro perspective. The Newton's cooling law and the energy conservation law are used in these two models orderly. And we draw the conclusion that the temperature has a linear correlation with time under the condition of small change in temperature. Secondly, we improve our model by taking natural cooling factor into consideration while injecting hot water. Moreover, on the basis of the analysis of the last step, we simplify our model. Then we use the optimal analysis method to find the best strategy on the foundation of our improved model. And the best strategy is to keep the best rate of injected water constantly. In this way, we can keep the temperature of water as close as possible to the comfortable temperature without wasting too much water only by adjusting the appropriate rate of the injected water.
\end{abstract}

\section{Introduction}

Imagine you drag your tired body home after a busy day, have a hot bath and then read a book on the sofa with clean and relax. Isn' t it an exciting thing in a day? However, the problem is that the bathwater will get cooler after a while, and you have to make your enjoy time interrupted to add hot water into the bathtub. But meanwhile you don' t want to waste too much water. In this case, we should propose a solution that makes sure you have a comfortable bath without paying excessive costs for water.

\section{Fundamental Assumptions}

No heat loss and no thermal power conversion in the system. And the system keep a dynamic balance in the heat exchange process.

-The bathroom is a closed system without ventilation so that we don' t take the impact of the wind in the cooling process into consideration.

The evaporation of water is not included in the calculating of water.

The temperature in the bathroom is constant, maintained at $25^{\circ} \mathrm{C}$; And the temperature of the injected water is constant.

The location of the faucet in the bathtub is fixed. To reduce water consumption, the faucet is fixed at the bottom of the bathtub.

- After entering the bathtub, the quality of the water in the bathtub stays the same 


\section{The traditional models about thermodynamics}

Table.1Parameter description

\begin{tabular}{ll}
\hline Parameter & Description \\
\hline$h$ & Convective heat transfer coefficient \\
$T_{1}$ & The water temperature of any time \\
$T_{r}$ & Indoor temperature \\
$A$ & Cross section area \\
$C$ & Specific heat \\
$m_{0}$ & The quality of the water in the bathtub \\
$T_{0}$ & The initial temperature \\
$T_{e}$ & The temperature of water when it starts to cool \\
$q_{1}$ & The flow rate of the injected water,the quality of injection water \\
$T_{s}$ & per second \\
$\Delta q$ & The target temperature \\
$T$ & The speed difference between the inflow and outflow water \\
$m_{1}$ & The temperature of the injection water \\
$m_{2}$ & The quality of injection water \\
\hline
\end{tabular}

The temperature of the bathtub water in space and time is solved by using three traditional mathematical models, a model of nature cooling, a model of injecting hot water, and a model of the water temperature distribution in the bathtub. The first two models describe the temperature in the bathtub in time from a macro perspective. The temperature distribution of the water in bathtub shows the temperature in the bathtub in space from a micro perspective.

Newton gave a description on the decrease rate of the object 's temperature with the law of cooling. And it reveals that when the object' $s$ temperature is higher than the surrounding media' $s$, it will transfer heat to others and cool down following the law. When there is temperature difference between the surface and the surrounding, the heat loss from the unit area per unit time is proportional to the difference, and the proportional coefficient is called heat transfer coefficient[1]. It can be described as follows:

$$
\frac{d Q}{d S d t}=h\left(T_{1}-T_{r}\right)
$$

Make integration on the cross section of heat conduction,

$$
\frac{d Q}{d t}=A h\left(T_{1}-T_{r}\right)
$$

According to the formula of heat calculation, when the temperature changed from $T_{0}$ to $T_{1}$, the heat emitted $Q=c m_{0}\left(T_{0}-T_{1}\right)-c m_{0} \frac{d T_{1}}{d t}=A h\left(T_{1}-T_{r}\right)$ After derivation calculus to $\mathrm{t}$, we can get

Thus $T_{1}=e^{k t+s}+T_{r}$, Where $k=-\frac{A h}{c m_{0}}$, s is a constant. We assume the temperature when it starts to cool is $T_{e}$, and we can determine the value of based on $\mathrm{s}$ initial condition, that is $s=\ln \left(T_{e}-T_{r}\right)$ Thus, the temperature of the water at any time when it naturally cool can be described as follows: $T_{1}=e^{k t}\left(T_{e}-T_{r}\right)+T_{r}$ Based on the formula of heat calculation, and the law of conservation of energy, we can obtain the equation of the heat exchange on a macroscopic level: $c m_{1}\left(T_{2}-T\right)=-c\left(m_{0}+\Delta q \bullet t-m_{2}\right)\left(T_{2}-T_{1}\right)$ [2]. We approximately simplifies the equation, and it turns to: $\left(m_{0}+\Delta q \bullet t\right) \Delta T_{1} / \Delta t+q_{1} T_{1}=q_{1} T$. Assume $\Delta t \quad$ tends to infinity and we can get the following 
differential equation after simplifying. $T_{1}+\frac{q_{i}}{m_{0}+\Delta q \bullet t} T_{1}=\frac{q_{i}}{m_{0}+\Delta q \bullet t} T$. Solving the differential equation, when the water temperature reaches its expected value, namely $T_{1}=T_{s}$, the time of injecting hot water is: $t_{o n}=\frac{m_{0}}{\Delta q}\left[\left(\frac{T_{s}-T}{T_{0}-T}\right)^{\frac{-q_{1}}{\Delta q}}-1\right] \quad\left(0<\frac{T_{s}-T}{T_{0}-T}<1\right)$

In this section,we assume that the initiative water in the bathtub has reached its capacity, so that the water inflow is equal to the water outflow when injecting hot water later. That is $q_{1}=q_{2}$, and $\Delta q=0$

Thus, the temperature of the water $T_{1}=\left(T_{0}-T\right) e^{\frac{-q_{1}}{m_{0}}}+T$, The time required for the water reaches its expected value $T_{s}$ turns into $t_{\text {on }}=-\frac{m_{0}}{q_{1}} \ln \frac{T_{s}-T}{T_{0}-T}$

\section{Improved model}

We state that the parameters we used in this section are consistent with the previous part.In the models above, we have established models to describe the change of water temperature over time. We consider the situations natural cooling and injecting hot water respectively. But in reality, cooling process also occurs when injecting hot water. Therefore, we improved our models by considering the two cases comprehensively. In order to make sure the temperature as close as possible to the initial temperature, we set the fluctuation range of one degree Celsius.

We assume the faucet is turned off when the temperature reaches $T_{0}+1$, and turned on when the temperature reaches its lower limit $T_{0}$.We know from the model of Nature cooling that $T_{1}=e^{k t}\left(T_{e}-T_{r}\right)+T_{r}$, and combined with the conditions above, we letbe $T_{1}=T_{0}, T_{e}=T_{0}+1$. Thus, we can obtain the reducing time required for one degree Celsius: $t_{\text {off }}=\frac{1}{k} \ln \frac{T_{0}-T_{r}}{T_{0}+1-T_{r}} t_{\text {on }}=-\frac{m_{0}}{q_{1}} \ln \frac{T_{s}-T}{T_{0}-T}$, when $T_{s}=T_{0}+1, t_{\text {on }}=-\frac{m_{0}}{q_{1}} \ln \frac{T_{0}+1-T}{T_{0}-T}$ We consider the influence of cooling while injecting hot water. We know the rising rate of temperature is $\frac{1}{t_{o n}}$ without consideration of cooling, and the reducing rate is $\frac{1}{t_{\text {on }}}$ when only consider the cooling process. we superpose temperature and time two factors and the required time for rising one degree becomes $t_{\text {on }}^{\prime}=\frac{1}{\frac{1}{t_{\text {on }}}-\frac{1}{t_{\text {off }}}}=\frac{t_{\text {on }} \bullet t_{\text {off }}}{t_{\text {off }}-t_{\text {on }}}$. Therefore, we can obtain a set of equations about the water temperature:

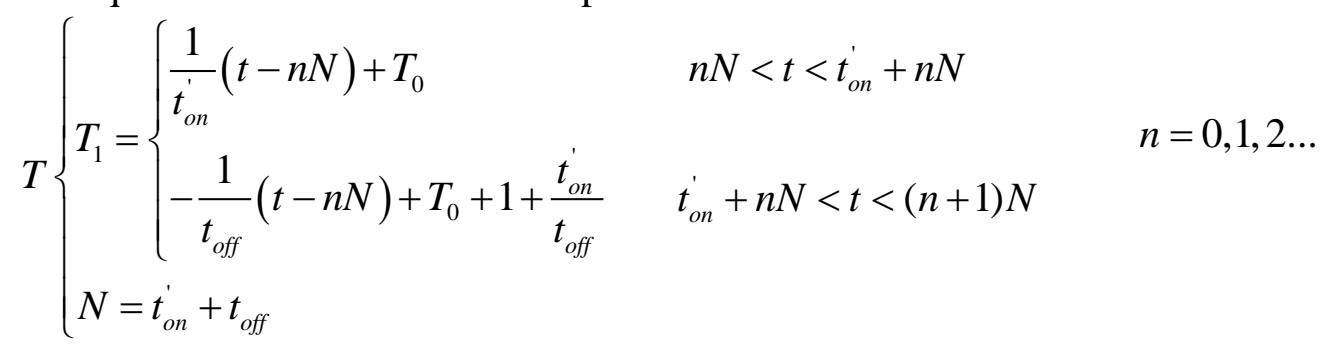




\section{Determine the best strategy}

Let the total time to take a bath be $\mathrm{Z}$, the total consumption of water be $\mathrm{D}$, then $D=\frac{Z}{N} t_{o n}^{\prime} \bullet q_{1}$, and the consumption per unit time is $f=\frac{D}{Z}=\frac{t_{\text {on }}^{\prime} \bullet q_{1}}{t_{\text {on }}^{\prime}+t_{\text {off }}}$ As the total time to take a bath differ from man to man, and it' s too difficult to evaluate, we only analyze the consumption per unit time, and our goal is to seek the minimum of $\mathrm{f}$. And the constraint conditions are : $\left\{\begin{array}{l}40^{\circ} \mathrm{C} \leq T \leq 45^{\circ} \mathrm{C} \\ 0<q_{1} \leq 0.3 \\ 36^{\circ} \mathrm{C} \leq \mathrm{T}_{0} \leq 42^{\circ} \mathrm{C}\end{array}\right.$ In the case of injecting hot water discontinuously, the rising rate of water temperature should not be lower than the reducing rate in a cycle, that means $t_{\text {on }}-t_{\text {off }} \leq 0$, solve it and we get $q_{1} \geq \frac{A h}{c} \frac{\ln \frac{T_{0}+1-T}{T_{0}-T}}{\ln \frac{T_{0}-25}{T_{0}-24}}=q$ After simplified the formula, we can find that the consumption of water per unit time is independent of $q_{1}$ any more. We assume the water in the bathtub. Find the optimal solution by computer. Letbe $q_{1}=0.3$

Here

$\begin{array}{cc}T_{0} & 36.0000 \\ T & 45.0000 \\ A & 1.0500 \\ h & 200.0000 \\ t_{\text {on }}^{\prime} & 73.6780 \mathrm{~s} \\ t_{\text {off }} & 400.2523 \mathrm{~s}\end{array}$

The initial temperature of water also represents a person's requirement to the water temperature. We can see from the data that when the water temperature requirements to achieve the minimum value, the maximum value of hot water temperature, the water consumption is the least.

\section{Summary}

Through the analysis above, we can draw a conclusion that there are two strategies that we can take to make the water consumption achieve the optimal. Keep $q_{1}=q$, the temperature in the bath can be maintained without controlling the faucet. Let $q_{1}>q$, the temperature in the bath varies in one degree Celsius by controlling the time that faucet turns on.Through the comparative analysis of the above two strategies, we can find that the first one is more convenient. And if we take the temperature influence on the $\mathrm{k}$ into consideration, the first strategy is prior to the second one. Therefore, the first strategy is the best strategy that we find to make the water consumption achieve its optimal.

\section{Reference}

[1] Chen Zhihua, Wang Darui, Liu Qiujuan, et al. Design of water temperature control model for sea cucumber aquaculture[J]. computer engineering and application, 2007,43 (32): 225-229.DOI:10.3321/j.issn:1002-8331.2007.32.066. 
[2] Zhao Jianqiang, Sun Yun Lou, Jia Min Qin. Plates of different shapes in the electric oven heating of the heat distribution mathematical model and numerical simulation [J] . Xinxiang University Journal (NATURAL SCIENCE EDITION ), 2013, (4) : $249-25125$ 3. D O I : 10 . 3 969 / j . iss n. $1674-3326.2013 .04 .004$. 